Sports Science

\title{
Reproducibility of an endurance test for master swimmers
}

\author{
Ezequiel Soares da Silva ${ }^{1}$ (D), Francivaldo Santos da Silva ${ }^{2}$ (D), Ivana Alice Teixeira Fonseca ${ }^{1}$ (D), \\ Marcelo Henrique Alves Ferreira da Silva ${ }^{2}$ (D), Maria Irany Knackfuss ${ }^{1}$ (D), \\ Glêbia Alexa Cardoso ${ }^{1}$ (D), Tiago Manoel Cabral dos Santos Barbosa ${ }^{3,4}$ (D), \\ Adalberto Veronese da Costa $^{1}$ \\ ${ }^{1}$ Universidade do Estado do Rio Grande do Norte, Departamento de Educação Física, Programa \\ de Pós-Graduação em Saúde e Sociedade, Mossoró, RN, Brazil; ${ }^{2}$ Universidade do Estado do Rio \\ Grande do Norte, Departamento de Educação Física, Mossoró, RN, Brazil; ${ }^{3}$ Instituto Politécnico \\ de Bragança, Bragança, Portugal; ${ }^{4}$ Centro de Investigação em Desporto, Saúde e \\ Desenvolvimento Humano, Vila Real, Portugal.
}

\begin{abstract}
Associate Editor: Gustavo R da Mota (D), Universidade Federal do Triângulo Mineiro, Uberaba, MG, Brazil. E-mail: grmotta@gmail.com.
\end{abstract}

\begin{abstract}
Aim: This study aimed to verify the reproducibility of an endurance test for master swimmers. Methods: Seventeen male swimmers, aged from 30 to 50 years $(35.06 \pm 5.36)$, weight $(78.71 \pm 13.06 \mathrm{~kg})$, height $(174.35 \pm$ $0.07 \mathrm{~cm}$ ), participated in this study. The participants underwent the Progressive Swim Test (PSwT), which corresponds to swimming progressively to exhaustion in a 25 -meter pool, guided by the recording of sound signals indicating the swimming rhythm. We verified the following variables: heart rate before the test (BHR) and right after the end of the test (AHR), followed by the rate of perceived exertion (RPE) and a total of laps performed ( $S N$ - swimming number). For comparison between the three days of tests, the one-way repeated measures ANOVA with Tukey's Post-Hoc was performed. Relative reproducibility was performed by the $r$ test and the intraclass correlation coefficient (ICC). Absolute reproducibility was determined using the coefficient of variation (CV) and the confidence interval (CI). Results: The results showed high reproducibility in (PSwT) in master swimmers in the analyzed variables of intergroup and intragroup with relative reproducibility of $r>0.60$ and ICC $>0.70$ and absolute reproducibility with CI $95 \%$ (lower -1.10 and upper 0.99 ) and CV $<10 \%$. Most of the cases were within the limits of agreement of the Bland-Altman tests, all variables were considered a level of significance with $(\mathrm{p}<0.05)$. Conclusion: The Progressive Swim Test (PSwT) is a reproducible instrument in the assessment of endurance in master swimmers.
\end{abstract}

Keywords: swimming, physical evaluation, resistance training, performance.

\section{Introduction}

Swimming is a physical exercise that provides numerous health benefits. When performed regularly, it causes significant decreases in systolic and diastolic pressures, improvement of glycemic control, cardiorespiratory and musculoskeletal conditions, as well as, the general health status of swimmers ${ }^{1-3}$. It is a motor activity composed of various contexts and objectives, adding the competitive and non-competitive sport, leisure, and health, and can be practiced by different age groups ${ }^{4}$. Swimming is a regular and systematic aerobic exercise. It presents as a measure of prevention and treatment against the impairment on the body's systems, improving the quality of life of swimmers ${ }^{5,6}$. The diagnosis of health status by the assessment of cardiorespiratory fitness is an aspect of great value when it is intended to measure the risks of cardiovascular diseases and the increase in life expectancy ${ }^{7,8}$.
However, the search for training programs for competitive and non-competitive purposes by individuals over 35 years of age has become increasingly common ${ }^{9}$. Master swimmers show increasing life expectancy, similar to other middle-aged people who engage in regular physical exercise, considering with this sport the possibility of participating in competitions over several years, however, it is necessary to monitor their health conditions and control the training loads periodically through physical tests ${ }^{10,11}$. Physical assessments are essential, due to the possibility of obtaining the subject's real fitness level and, thus, designing customized exercise prescriptions, either for health or performance purposes ${ }^{12,13}$. For swimming, it is believed that physical tests that increase the intensity over certain distances, would be the most suitable for working with swimmers who cannot control different swimming rhythms ${ }^{14,15}$. Thus, swimming tests in progressive series 
are those where submaximal efforts occur throughout the test until fatigue is reached ${ }^{16}$.

The swimming tests are increasingly adhering to specificity principles by various athletes, and they are no longer exclusively for high-performance counterparts. It is worth noting that a test needs to be valid and reproduced in different groups or cohorts by the same evaluator or different evaluators ${ }^{17}$. The Progressive Swim Test (PSwT), an exclusive test for 18 to 30 years old male swimmers non-participants in high-performance competitions, presented a level of favorable reproducibility and validity with possible application in the indirect assessment of aerobic conditioning for the prescription of non-competitive swimming ${ }^{18-21}$.

In this sense, the specific tests for master swimmers still need to be studied, developed, or adapted to existing protocols. The PSwT can evaluate these swimmers for starting with relatively low intensity and can be an alternative of indirect evaluation that meets the different categories of master swimming. However, it is necessary to verify if this method would be reproducible for this audience. Therefore, this study verified the reproducibility of the endurance test for master swimmers. It was hypothesized that the results are highly reproducible.

\section{Methods}

\section{Experimental approach to the problem}

This comparative descriptive study was carried out in three days, with 48-hour intervals, the first and second days were evaluated by examiner $\mathrm{A}$ and the third day by examiner $\mathrm{B}$. This control procedure regarding the intervals between assessments was important so that one test does not interfere with the others ${ }^{22,23}$. The protocol selected was the Progressive Swim Test (PSwT). In this test, the participants progressively swim to exhaustion in a $25 \mathrm{~m}$ pool, guided by sound signals, using a Swim MP3 device indicating the swimming rhythm. After the test start signal, which has a determined time of 28 " 30 to perform the first lap, there is a partial time reduction of 1 second after two laps performed. ${ }^{19}$. All swimmers were of regional level and affiliated to the master federation of the state of origin.

Body composition and the first test session with PSwT were performed on different days, was verified the following variables were: the heart rate before the test (BHR) and right after the end of the test (AHR), followed by the rate of perceived exertion (RPE) and a total of laps performed ( $S N$ - swimming number) as shown in (Figure 1). In order to establish the estimated overall efficiency of each swimmer, time measurements and stroke counts for each lap were taken ${ }^{24}$.

\section{Subjects}

The sample comprised 17 male swimmers, aged from 30 to 50 years $(35.06 \pm 5.36$ years), weight $(78.71 \pm$ $13.06 \mathrm{~kg})$, height $(174.35 \pm 0.07 \mathrm{~cm})$, arm span $(180.47 \pm$ $0.08 \mathrm{~cm})$, body fat percentage $(20.39 \% \pm 5.89 \%)$. Participants in this study were those who presented a proficient front crawl swimming technique, trained at least three times a week and were able to swim continuously at least $800 \mathrm{~m}$ per session. Exclusion criteria included swimming athletes who were in training for national and international competitions, as well as those who indicated any type of disease that made it difficult to perform the test (for example flu, fever, or any type of injury). The reproducibility test was evaluated by two swimming coaches who had a minimum of 10 years of background experience, either as a coach or swimmer.

The study was approved by the Human Research Ethics Committee of the State University of Rio Grande do Norte, CAAE: 85371918.0.0000.5294, opinion No. 2.591.976. All participants signed the Free and Informed Consent Form, meeting the requirements of the National Health Council, according to resolution 466/12.

\section{Procedures}

Before testing days, participants were briefed on the protocol details, they were also instructed to refrain from having any type of food supplementation, caffeine, or alcoholic beverages, as well as to avoid physical exercise 24 hours before the tests. The swimmers followed the same order of evaluation on all testing days.

Familiarization with the test, procedures, and the Borg scale occurred in three stages. First, a video was presented showing the execution of the test to the evaluated. Second, the practical demonstration of the test with an experienced swimmer. Third, a series between 50 and $100 \mathrm{~m}$ with Swim MP3 was performed as a warm-up and further familiarization with the timing of the beeps. The test started when the swimmer stood in the pool, with part of his body leaning against the start edge waiting for the following announcement: "Attention swimmer, prepare for the test". Soon after, a short beep accompanied by a visual signal from the evaluator's hands marked the beginning of the test.

During the test, to facilitate the identification of the correct rhythm of progression for each lap, the PSwT presents a long beep of a different type of sound to inform the swimmer that he should be near or in the middle of the pool. After each completed lap, another short beep was given to indicate the start of the next lap, and so on until exhaustion. The swimmer was instructed to keep to the rhythm of the beeps from the Swim MP3 in order to always be within the beginning or end of the 5-m zone when the short beep would be heard. As identifiers for swimmers and evaluators, cones were placed along the 

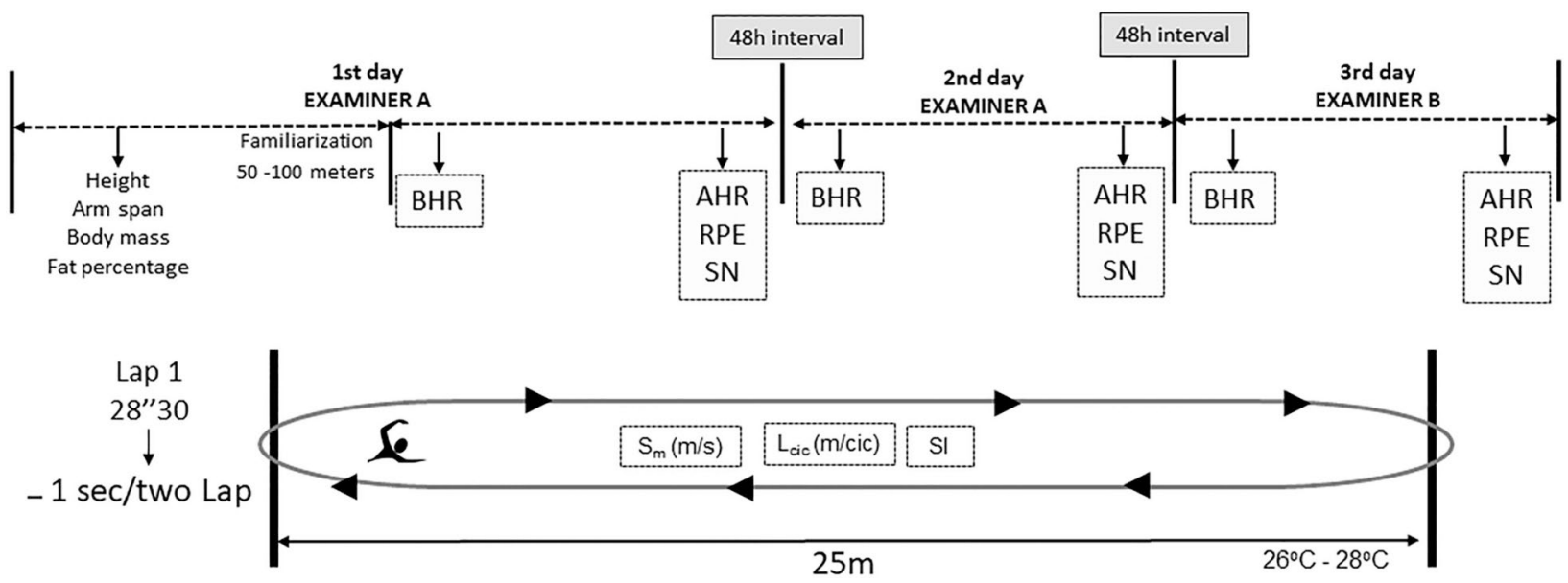

Figure 1 - Schematic illustration of study design. BHR - before (test) heart rate in beats per minute; AHR - after (test) heart rate in beats per minute; $S N$ swimming number (laps); $S_{m}$ - Average speed in meters per second (average number of laps performed); $L_{c i c}$ - stroke cycles length in meters per cycle (average number of laps performed); $S I$ - stroke index.

edge, and $5 \mathrm{~kg}$ rings inside the pool, to mark the initial five meters, the middle of the pool, and the final five meters. The evaluators were allowed to monitor the lap count giving verbal and visual signals so that the swimmers could maintain the progressive test pace. The test ended when the swimmer was unable to reach the five-meter zone preceding the edge of the pool twice. AHR, RPE, and $S N$ were measured using the swimmer's head as part of the reference body.

To quantify the time and stroke count for each lap, a video recording was made through a camera positioned outside and in the center of the pool, at a distance of approximately $15 \mathrm{~m}$ from the test streak. The execution of the $15 \mathrm{~m}$ of each lap was analyzed, excluding the initial and final $5 \mathrm{~m}$ to eliminate any type of advantage related to edge thrusts and turns. Thus, the mathematical equations used were ${ }^{24,25}$ :

$$
S_{m}=\frac{R}{t}
$$

where $S_{m}$ is the average speed, $R$ is the distance of the $15 \mathrm{~m}$ swam, and $t$ is the execution time.

$$
L_{c i c}=\frac{R}{N_{c i c}}
$$

Considering $L_{c i c}$ the length per cycle, $R$ the distance of the $15 \mathrm{~m}$ swam, and $N_{c i c}$ the number of stroke cycles equivalent to two strokes.

$$
S I=L_{c i c} \times S_{m}
$$

The product between the $L_{c i c}$ and the $\mathrm{S}_{m}$ corresponds to the SI Stroke Index, that is, the higher the $S I$, the better the swimmer's efficiency.
Before each test the water temperature was measured to identify any difference in environmental conditions (Bestway ${ }^{\circledR}$ Floating Pool Thermometer, accurate to $1{ }^{\circ} \mathrm{C}$; Bestway Inflatables North America Inc, Mississauga, $\mathrm{ON}$, Canada), the temperature ranged from $26^{\circ} \mathrm{C}$ to $28^{\circ} \mathrm{C}$.

To assess the body composition of the swimmers, the height (Sanny® wall stadiometer, range from 80 to $220 \mathrm{~cm}$ - American Medical do Brasil Ltda), arm span (Trenna Sanny®, range from 0 to $200 \mathrm{~cm}$ - American Medical do Brasil Ltda.), body mass, and fat percentage (digital scale of hand-to-foot bioelectrical impedance analysis, Omron ${ }^{\circledR}$ brand, model HBF-514C - Kyoto, Japan).

For the transmission of the beep recording sound during the test, the SwiMP3 v2 device (Finis ${ }^{\circledR}$ Inc, Livermore, CA) was attached to the silicon strip of the swimmer's goggles, which allows the sound to be transferred by bone conduction providing good sound clarity when submerged in water.

For the analysis of the time and the stroke count in $15 \mathrm{~m}$ of each lap in the pool during the test, a Swann Freestyle HD Action Camera (Swann®, New Jersey, USA) was used, with 1080p high-definition video at $30 \mathrm{fps}$ (1920 $x$ 1080) which allows the capture of images without errors $^{26,27}$.

The BHR and AHR were measured using a Polar ${ }^{\circledR}$ FT1 measurement interval of 5 seconds. This equipment has a frequency transmission of $5 \mathrm{kHz}$, heart rate measurement accuracy of $\pm 1 \%$ or $\pm 1 \mathrm{bpm}$, in stable conditions, operates at a temperature of $-10{ }^{\circ} \mathrm{C}$ to $+50^{\circ} \mathrm{C}$ and has a measurement range and heart rate limit of 15 to $240 \mathrm{bpm}$ and 30 to $199 \mathrm{bpm}$, respectively (2013 Polar Electro Oy, Professorintie 5, FIN 90440 KEMPELE, Finland, www.polar.com). For this measure, the swimmers were standing inside the pool with water at the swimmer's chest level, avoiding any variation between land and water environment. 
For the RPE, where it was verified at the end of the test, the modified Borg's RPE score (scale from 0 to 10) proposed for swimming was used, considering 0 as "Rest" and 10 as "Maximal"28. The $S N$ was verified right after the RPE evaluation considering the middle of the pool as a reference for rounding the quantity, which was also checked by the video recording of the test days.

\section{Statistical analysis}

Descriptive values were expressed as mean and standard deviation. For the normality and homogeneity of the data, the Shapiro - Wilk and Levene tests were applied respectively. For comparison between the three days of tests, the ANOVA One Way of repeated measures with Tukey's Post-Hoc was performed. For the reproducibility analysis, measurements from the 1st and 3rd days (Examiner A $x$ Examiner B) were used as intergroup and measurements from the 1st and 2nd days (Examiner A x Examiner A) as intragroup. To analyze relative reproducibility, the $r$-test was used (Pearson's linear correlation), following the classification according to Hopkins ${ }^{29}$. As well as the intraclass correlation coefficient (ICC) categorized as not acceptable $(<0.70)$, acceptable $(0,70-0.79)$, good agreement (0.80-0.89), and excellent agreement ( $>0.90)$, and absolute reproducibility was determined using the Coefficient of Variation (CV) and the Confidence Interval $(\mathrm{CI})^{30,31}$.

For graphical analysis of the variable's orders of magnitude, the Bland - Altman plot was applied to establish the limits of agreement. All variables were considered significant when $\mathrm{p}<0.05$. Data were analyzed using the statistical packages SPSS for Windows (v 20; IBM Corporation, Armonk, NY) and MedCalc for Windows (v 19.3.1; MedCalc Software BVBA, Mariakerke, Belgium).

\section{Result}

Table 1 highlights the descriptive values in which they were expressed as mean and standard deviation com-

Table 1 - Descriptive values on different test days applied to master swimmers.

\begin{tabular}{lccc}
\hline Variable & 1st day & 2nd day & 3rd day \\
\hline BHR $(\mathrm{bpm})$ & $77.93 \pm 8.87$ & $80.56 \pm 9.67$ & $78.67 \pm 8.44$ \\
AHR $(\mathrm{bpm})$ & $157.4 \pm 11.50$ & $161.3 \pm 10.90$ & $163.8 \pm 9.90$ \\
$S N$ & $10.7 \pm 4.10$ & $10.1 \pm 3.90$ & $10.5 \pm 3.90$ \\
$S_{m}(\mathrm{~m} / \mathrm{s})$ & $0.96 \pm 0.05$ & $0.98 \pm 0.05$ & $0.98 \pm 0.04$ \\
$L_{c i c}(\mathrm{~m} / \mathrm{cic})$ & $1.82 \pm 0.10$ & $1.85 \pm 0.15$ & $1.83 \pm 0.15$ \\
$S I$ & $1.85 \pm 0.15$ & $1.86 \pm 0.30$ & $1.87 \pm 0.27$ \\
\hline
\end{tabular}

Abbreviations: BHR - before (test) heart rate in beats per minute; AHR after (test) heart rate in beats per minute; $S N$ - swimming number (laps); $S_{m}$ - Average speed in meters per second (average number of laps performed); $L_{c i c}$ - stroke cycles length in meters per cycle (average number of laps performed); $S I$ - stroke index. paring the days, considering that there were no significant intergroup and intragroup differences in the analyzed variables.

Table 2 shows the values applied to the master swimmers. The highlight for relative reproducibility of $r>0.60$ (intergroup) and ICC $>0.70$ (intragroup), and absolute reproducibility with $\mathrm{CI}$ between lower -1.10 and upper 0.99 (intragroup), and a $\mathrm{CV}<10 \%$ (intergroup) for variables with significance.

Figure 2 shows the intragroup limits of agreement for the variables analyzed. It was found that most cases were within the upper and lower limits of the Bland-Altman graphs.

\section{Discussion}

This study aimed to verify the reproducibility of an endurance test for master swimmers. The results showed high reproducibility in the PSwT in the variables analyzed when applied to master swimmers on different days by different evaluators, considering the same analysis conditions performed in young adult swimmers ${ }^{18-20}$, however, the values obtained were lower than the findings in this study.

It was observed that the master swimmers started the test with a BHR below $90 \mathrm{bpm}$, that is, with their rest frequency or close to it. Additionally, they did not show variability in heart rate, characterizing that those evaluated did not make efforts the day before that would compromise performance in the test. The test was performed by swimmers intensely, in agreement with the values obtained in the subjective perception of effort, which was classified as "Maximal" in the 3 days of tests. Considering that the RPE has not reached the last stage, this fact can be corroborated in the difficulty of the individuals to interpret the scale at the end of the test. Although the RPE has a good validity in the intensity classification ${ }^{28,32}$. However,

Table 2 - Intergroup and intragroup values applied to master swimmers.

\begin{tabular}{lccccc}
\hline Variable & $\boldsymbol{r}$ & $\boldsymbol{p}$ & $\mathbf{C V}$ & ICC (95\% IC) & $\boldsymbol{p}$ \\
\hline BHR $(\mathrm{bpm})$ & 0.22 & 0.45 & $6 \%$ & $0.31(-1.10 ; 0.76)$ & 0.25 \\
AHR $(\mathrm{bpm})$ & 0.42 & 0.14 & $5 \%$ & $0.75(0.99 ; 0.91)$ & $0.00 *$ \\
$\mathrm{RPE}$ & 0.68 & $0.01 *$ & $3 \%$ & $0.77(0.35 ; 0.92)$ & $0.00 *$ \\
$S N$ & 0.93 & $0.00 *$ & $8 \%$ & $0.96(0.89 ; 0.99)$ & $0.00 *$ \\
$S_{m}(\mathrm{~m} / \mathrm{s})$ & 0.67 & $0.01 *$ & $2 \%$ & $0.74(0.30 ; 0.91)$ & $0.00 *$ \\
$L_{c i c}(\mathrm{~m} / \mathrm{cic})$ & 0.53 & 0.06 & $4 \%$ & $0.84(0.52 ; 0.95)$ & $0.00 *$ \\
$S I$ & 0.81 & $0.00 *$ & $5 \%$ & $0.74(0.25 ; 0.91)$ & $0.01 *$ \\
\hline
\end{tabular}

Abbreviations: $r$ - Pearson correlation coefficient; ICC - intraclass correlation coefficient; IC - confidence interval; CV - coefficient of variation; BHR - before (test) heart rate in beats per minute; AHR - after (test) heart rate in beats per minute; RPE - rate of perceived exertion; $S N$ - swimming number (laps); $S_{m}$ - Average speed in meters per second (average number of laps performed); $L_{c i c}$ - stroke cycles length in meters per cycle (average number of laps performed); SI - stroke index. *Significant difference $\mathrm{p}<0.05$. 

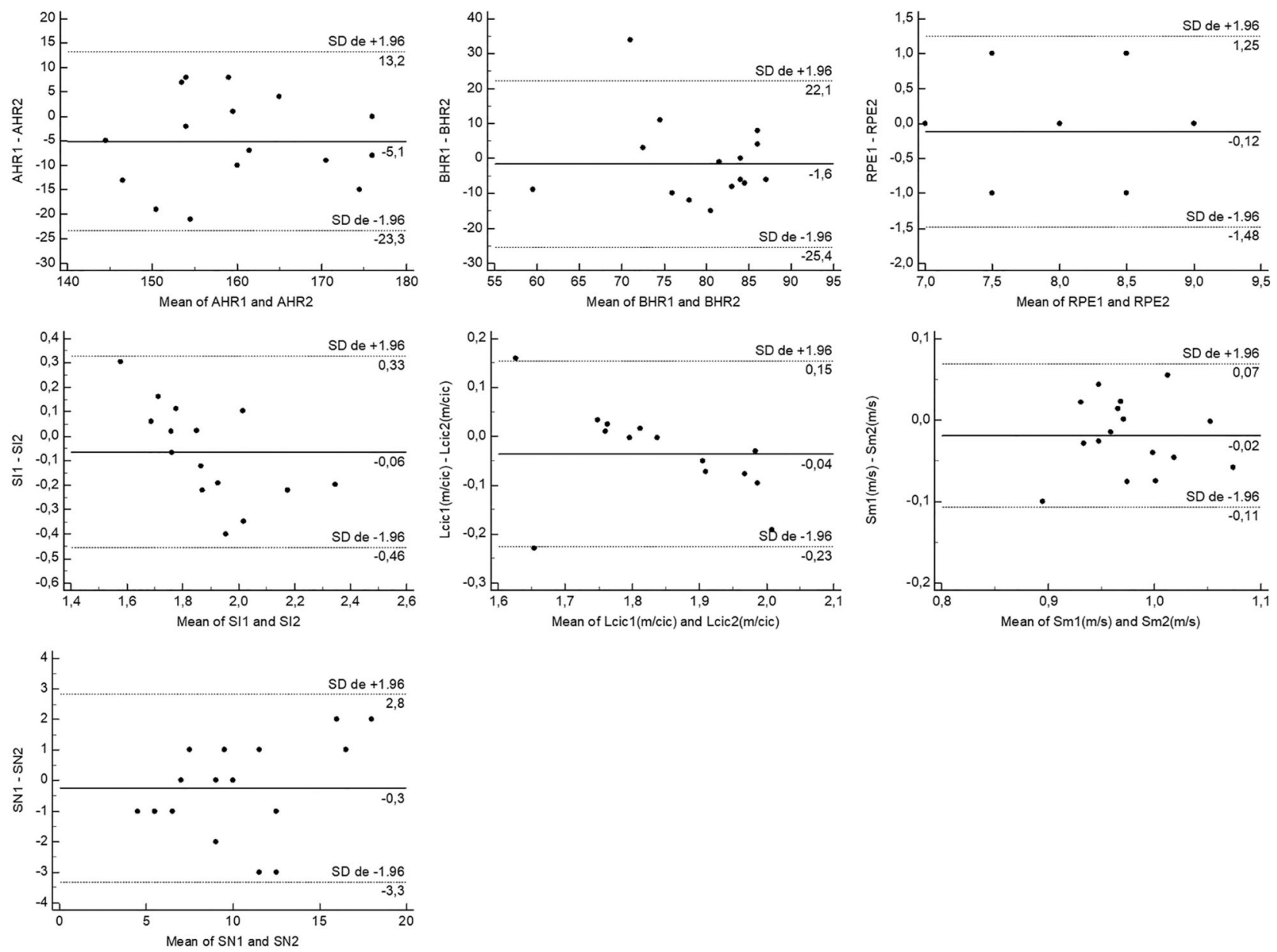

Figure 2 - Limits of intragroup agreement in master swimmers. BHR - before (test) heart rate in beats per minute; AHR - after (test) heart rate in beats per minute; $S N$ - swimming number (laps); $S_{m}$ - Average speed in meters per second (average number of laps performed); $L_{c i c}$ - stroke cycles length in meters per cycle (average number of laps performed); $S I$ - stroke index.

it was observed that the subjects reached the state of fatigue for not being able to follow the swimming rhythm proposed by the test beeps.

Regarding the $S N$, even though there were no significant differences, it was observed that there was a greater dispersion in the results on the first day because of familiarization with the PSwT. The first test day could be a stimulus for swimmers to overcome $S N$ in the following days ${ }^{18}$. Regardless of the experimental manipulation, psychological factors can affect the amount of work that an athlete can perform, thus significant impacts on performance are observed ${ }^{33}$. In open exercises where a specific goal and endpoint are not established, the greater the influence for the swimmer to reach a state of exhaustion faster compared to a test where the endpoint is determined, as the motivation factor will be higher. ${ }^{34}$ Therefore, the greater the adaptation throughout the training, the more consistent the test will become.

As for the similarities found in the average values of $S_{m}, L_{c i c}$, and $S I$, demonstrate a standardization in the bio- mechanics of movement between days, even the test was performed using an intensity progression determined by the time at each lap in the pool. It is necessary to consider that for better propulsive efficiency of the swim, the shorter the time in the execution in a given distance with the greater the length of the strokes, the greater the SI, consequently a swimmer who offers a better biomechanical use in his swim performance, the more efficient it will $\mathrm{be}^{25,35}$.

By comparing the results between the days and different evaluators, the relative reproducibility obtained significant results, especially the variables RPE, $S N$, and $S I$. In previous studies to verify the reproducibility and applicability of PSwT in non-expert young adult swimmers, significant results regarding relative reproducibility were also obtained for RPE and $S N,(r>0.50)$ and $(r>0.90)$ respectively ${ }^{18,19}$. However, these results were inferior to the findings in this study. Thus, these variables were considered determinants of the swimmers' performance in the test. The absolute reproducibility in all vari- 
ables was considered acceptable, with $\mathrm{CV}$ being less than $10 \%{ }^{30}$.

Figure 2 presents graphically the conditions of the intragroup of magnitude for SI. Thus, the $S N$ variable favored the confirmation of good reproducibility. Average speed is a useful measure to prove swimming performance. If the swimmer does not have a good stroke length, a higher level of stroke frequency will be required to achieve satisfactory performance. Therefore, increases or decreases in the average speed are determined by a combination of increases or decreases in frequency and stroke length $^{36,37}$. The decline manifests the development of local fatigue, reducing the production of mechanical energy and, consequently, performance ${ }^{25}$. Only two swimmers were beyond these limits in the $L_{c i c}$ variable. An explanation for this result might be the occurrence of greater variations in the swimmer's impulse throughout the stroke cycle in each technique performed. These intracyclic impulse variations result in additional mechanical work and, consequently, a greater total energy expenditure ${ }^{25}$.

Overall, it was shown that $S_{m}$, for both intergroup and intragroup variations, was a determining condition for establishing the propulsive efficiency of the swimmer. This variable was essential for $S I$ to obtain good intergroup reproducibility. Because when the length of the cycle is not measured, the average speed is presented as a decisive variable to attest to the efficiency of a swimmer $^{25,38,39}$.

This study presents the following limitations: The impossibility of more intensive training of swimmers to respond more accurately to RPE and the impossibility of performing measurements of intracyclic impulse variations and total energy expenditure.

\section{Conclusion}

In conclusion, variables showed that the PSwT is a reproducible instrument to be applied in the assessment of endurance in master swimmers. This test presents a lowcost proposal to assess the endurance of master swimmers, considering the reality and specificity of their practitioners.

\section{Acknowledgments}

This study was supported by The Coordination for the Improvement of Higher Education Personnel (CAPES). The participants who enrolled in this study and the Laboratory for the Assessment of Aquatic Performance.

\section{References}

1. Conti AA. Swimming, physical activity, and health: a historical perspective. Clin Ter. 2015;166(4):179-82. doi
2. Igarashi Y, Nogami Y. The effect of regular aquatic exercise on blood pressure: a meta-analysis of randomized controlled trials. Eur J Prev Cardiol. 2017;25(2):190-9. doi

3. Nualnim N, Parkhurst K, Dhindsa M, Tarumi T, Vavrek J, Tanaka H. Effects of swimming training on blood pressure and vascular function in adults $>50$ years of age. Am J Cardiol. 2012;109(7):1005-10. doi

4. Escalante Y, Saavedra JM. Swimming and aquatic activities: state of the art. J Hum Kinet. 2012;32:5-7. doi

5. Agarwal SK. Cardiovascular benefits of exercise. Int J Gen Med. 2012;5:541-5. doi

6. Fiuza-Luces C, Santos-Lozano A, Joyner M, Carrera-Bastos P, Picazo O, Zugaza JL, et al. Exercise benefits in cardiovascular disease: beyond attenuation of traditional risk factors. Nat Rev Cardiol. 2018;15(12):731-43. doi

7. Kaminsky LA, Arena R, Ellingsen Ø, Harber MP, Myers J, Ozemek C, et al. Cardiorespiratory fitness and cardiovascular disease - the past, present, and future. Prog Cardiovasc Dis. 2019;62(2):86-93. doi

8. Mandsager K, Harb S, Cremer P, Phelan D, Nissen SE, Jaber W. Association of cardiorespiratory fitness with longterm mortality among adults undergoing exercise treadmill testing. JAMA Netw Open. 2018;1(6):e183605. doi

9. Brauer Júnior AG, Bento PCB. Analysis of master swimmers' sports performance for different freestyle distances. J Phys Educ. 2018;29(1):1-10. doi

10. Moreira CR, Nascimento Junior JRA, Mizoguchi MV, Oliveira DV, Vieira LF. Impact of adhesion reasons in the motivational regulation of master swimmers during the season. Rev Bras Cineantropom Hum. 2016;18(4):429-40. doi

11. Rubin RT, Lin S, Curtis A, Auerbach D, Win C. Declines in swimming performance with age: a longitudinal study of Masters swimming champions. Open access J Sport Med. 2013;4:63-70. doi

12. Costa MJ, Bragada JA, Mejias JE, Louro H, Marinho DA, Silva AJ, et al. Effects of swim training on energetics and performance. Int J Sport Med. 2013;34(6):507-13. doi

13. Silva I, Oliveira LS, Berenguer MF, Sousa AVF, Nascimento JA, Costa MC. Reproducibility of an effort protocol during deep-water running. Motricidade. 2010;6(4):47-54. doi

14. de Souza H, Alves ES, Ortega L, Silva A, Esteves AM, Schwingel PA, et al. Incremental exercise test for the evaluation of peak oxygen consumption in paralympic swimmers. J Sports Med Phys Fitness. 2015;56(4):368-75.

15. Pelarigo JG, Fernandes RJ, Ribeiro J, Denadai BS, Greco $\mathrm{CC}$, Vilas-Boas JP. Comparison of different methods for the swimming aerobic capacity evaluation. J Strength Cond Res. 2018;32(12):3542-51. doi

16. Olbrecht J, Madsen Ø, Mader A, Liesen H, Hollmann W. Relationship between swimming velocity and lactic concentration during continuous and intermittent training exercises. Int J Sport Med. 1985;6(2):74-7. doi

17. Morais JE, Costa MJ, Forte P, Marques MC, Silva AJ, Marinho DA, et al. Longitudinal intra- and inter-individual variability in young swimmers' performance and determinant competition factors. Motriz: J. Phys. Ed. 2014;20 (3):292-302. doi

18. Veronese da Costa A, Costa MC, Carlos DM, Guerra LMM, Silva AJ, Barbosa TM. Reproducibility of an aerobic endu- 
rance test for nonexpert swimmers. J Multidiscip Healthc. 2012;5:215-21. doi

19. Veronese da Costa A, Costa MC, Oliveira SFM, Albuquerque FL, Guimarães FJSP, Barbosa TM. Validation of an equation for estimating maximal oxygen consumption of nonexpert adult swimmers. Open access J Sport Med. 2013;4:19-25. doi

20. Veronese da Costa A, Costa MC, Gomes JLB, Guimarães FJSP, Barbosa TM. Applicability of an indirect VO2max test: its association with the 400 meters freestyle performance. Motriz: J. Phys. Ed. 2016;22(4):304309. doi

21. Ruibal-Lista B, Palacios-Aguilar J, Prieto JA, García SL. Validation of a new incremental swim test as a tool for maximum oxygen uptake analysis in lifeguards. Int $\mathrm{J}$ Aquat Res Educ. 2019;11(3):1-12. doi

22. Pedersen BK, Hoffman-Goetz L. Exercise, and the immune system: regulation, integration, and adaptation. Physiol Rev. 2000;80(3):1055-81. doi

23. Burke LM, van Loon LJC, Hawley JA. Postexercise muscle glycogen resynthesis in humans. J Appl Physiol. 2017;122 (5):1055-67. doi

24. Barbosa TM, Lima V, Mejias E, Costa M, Marinho D, Garrido $\mathrm{N}$, et al. Propulsive efficiency and non-expert swimmers performance. Motricidade. 2009;5(4):27-43. doi

25. Barbosa TM, Bragada JA, Reis VM, Marinho DA, Carvalho C, Silva AJ. Energetics and biomechanics as determining factors of swimming performance: Updating the state of the art. J Sci Med Sport. 2010;13(2):262-9. doi

26. Nakashima M, Hatakeyama G, Homma M, Ito K. Simulation analysis of lift in synchronized swimming. Journal of Aero Aqua Bio-mechanisms. 2013;3:51-6. doi

27. Soh J, Sanders R. The clues are in the flow: how swim propulsion should be interpreted. Sports Biomechanics. 2019;20(7):798-814. doi

28. Wallace LK, Slattery KM, Coutts AJ. The ecological validity and application of the Session-RPE Method for quantifying training loads in swimming. J Strength Cond Res. 2009;23(1):33-8. doi

29. Hopkins WG. Measures of reliability in sports medicine and science. Sport Med. 2000;30(1):1-15. doi

30. Atkinson G, Nevill AM. Statistical methods for assessing measurement error (reliability) in variables relevant to sports medicine. Sports Med. 1998;26(4):217-38. doi

31. Currell K, Jeukendrup AE. Validity, reliability, and sensitivity of measures of sporting performance. Sports Med. 2008;38(4):297-316. doi

32. Chen M, Fan X, Moe S. Criterion-related validity of the Borg ratings of perceived exertion scale in healthy indivi- duals: a meta-analysis. J Sports Sci. 2002;20(11):873-99. doi

33. Wilmore JH. Influence of motivation on physical work capacity and performance. J Appl Physiol. 1968;24(4):45963. doi

34. Toussaint HM, Carol A, Kranenborg H, Truijens MJ. Effect of fatigue on stroking characteristics in an arms-only 100-m front-crawl race. Med Sci Sports Exerc. 2006;38(9):163542. doi

35. Morais JE, Garrido ND, Marques MC, Silva AJ, Marinho DA, Barbosa TM. The influence of anthropometric, kinematic and energetic variables and gender on swimming performance in youth athletes. J Hum Kinet. 2013;39(1):203211. doi

36. Craig AB, Skehan PL, Pawelczyk JA, Boomer WL. Velocity, stroke rate, and distance per stroke during the elite swimming competition. Med Sci Sport Exerc. 1985;17 (6):625-34. doi

37. Hay JG, Guimaraes ACS, Grimston SK. A quantitative look at swimming biomechanics. Swim Tech. 1983;20(2):11-17.

38. Psycharakis SG, Cooke CB, Paradisis GP, O'Hara J, Phillips G. Analysis of selected kinematic and physiological performance determinants during incremental testing in elite swimmers. J Strength Cond Res. 2008;22(3):951-7. doi

39. Zamparo P, Dall'Ora A, Toneatto A, Cortesi M, Gatta G. The determinants of performance in master swimmers: a cross-sectional study on the age-related changes in propelling efficiency, hydrodynamic position, and energy cost of front crawl. Eur J Appl Physiol. 2012;112(12):3949-57. doi

\section{Corresponding author}

Adalberto Veronese da Costa. Universidade do Estado do Rio Grande do Norte. Av. Prof. Antônio Campos, s/n.

Bairro Costa e Silva. Mossoró, RN.

E-mail: adalbertoveronese@uern.br.

Manuscript received on May 8, 2021

Manuscript accepted on January 17, 2022

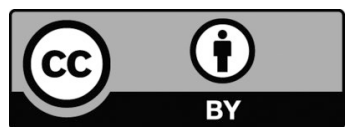

Motriz. The Journal of Physical Education. UNESP. Rio Claro, SP, Brazil - eISSN: 1980-6574 - under a license Creative Commons - Version 4.0 